\title{
Lymphatic Abnormalities in Noonan Syndrome Spectrum Disorders: A Systematic Review
}

\author{
Julia Sleutjes $^{\mathrm{a}}$ Lotte Kleimeier ${ }^{\mathrm{a}}$ Erika Leenders ${ }^{\mathrm{b}}$ Willemijn Klein ${ }^{\mathrm{c}}$ \\ Jos Draaisma ${ }^{a}$ \\ aDepartment of Pediatrics, Radboud University Medical Center, Radboud Institute for Health Sciences, Amalia \\ Children's Hospital, Nijmegen, The Netherlands; ${ }^{b}$ Department of Human Genetics, Radboud University Medical \\ Center, Nijmegen, The Netherlands; ' Department of Radiology and Nuclear Medicine, Radboud University Medical \\ Center, Nijmegen, The Netherlands
}

\section{Keywords}

Noonan syndrome spectrum disorders · Noonan syndrome · Genotype-phenotype relations · Lymphatic abnormalities

\begin{abstract}
Noonan syndrome spectrum disorders are a group of phenotypically related conditions, resembling Noonan syndrome, caused by germline pathogenic variants in genes within the Ras/mitogen-activated protein kinase (Ras/MAPK) signalling pathway. Lymphatic dysplasia with a clinical lymphatic abnormality is one of the major features. We performed a systematic review to get more insight in (1) the prevalence of clinically lymphatic abnormalities in patients with a genetically proven Noonan syndrome spectrum disorder, (2) if a genotype-lymphatic phenotype relation can be found and describe the clinical presentation and course of the lymphatic abnormality. Most studies report patients with Noonan syndrome. Prenatally, the prevalence of increased nuchal translucency differs from $7 \%$ in patients with pathogenic PTPN11 variants to $38 \%$ in patients with pathogenic RIT1 variants, and the prevalence of pleural effusions differed from $7 \%$ in patients with pathogenic SOS1 to $29 \%$ in patients with pathogenic RIT1 variants. Postnatally, the prevalence of lymphedema differs from $16 \%$ in patients with
\end{abstract}

karger@karger.com www.karger.com/msy

Karger $\stackrel{\text { ' }}{=}$
(C) 2021 The Author(s)

Published by S. Karger AG, Basel

This is an Open Access article licensed under the Creative Common Attribution-NonCommercial-4.0 International License (CC BY-NC) (http://www.karger.com/Services/OpenAccessLicense), applicable to the online version of the article only. Usage and distribution for commercial purposes requires written permission. pathogenic PTPN11 variants to $44 \%$ in patients with pathogenic SOS1 variants, and the prevalence of acquired chylothorax is $4 \%$ in patients with pathogenic RIT1 variants. Lymphatic abnormalities do occur in patients with cardiofaciocutaneous syndrome and Costello syndrome. In conclusion, Noonan syndrome spectrum disorders, Noonan syndrome in particular, are associated with lymphatic abnormalities. Combining the available published literature about genetically proven Noonan syndrome spectrum disorders, it appears likely that the lifetime prevalence of these abnormalities in Noonan syndrome is higher than the $20 \%$ that were generally accepted so far. This is increasingly important, because the activation of the RAS/MAPK pathway can be inhibited by RAS/MAPK inhibitors, and clinically severe lymphatic abnormalities may improve.

(C) 2021 The Author(s) Published by S. Karger AG, Basel

\section{Introduction}

Noonan syndrome spectrum disorders are a group of phenotypically related conditions, resembling Noonan syndrome (NS), caused by germline pathogenic variants in genes within the Ras/mitogen-activated protein kinase (Ras/MAPK) signalling pathway [Grant et al., 2018]. The 
most prevalent syndrome is Noonan syndrome (OMIM 163950). The cardinal features are characteristic facies, short stature, congenital heart defects, cryptorchism in boys, and lymphatic dysplasia [van der Burgt, 2007]. The clinical presentation however can be extremely variable. Activation of the Ras/MAPK pathway is considered to cause abnormal development of several tissues, resulting in birth defects [Tajan et al., 2018]. Other Noonan syndrome spectrum disorders are Noonan syndrome with multiple lentigines (NSML; OMIM 151100), Noonanlike syndrome with loose anagen hair (NS-LAH; OMIM 607721), cardiofaciocutaneous syndrome (CFCS; OMIM 115150) and Costello syndrome (CS; OMIM 218040) [Tajan et al., 2018]. There are pathogenic variants in different genes that can cause NS. Currently, at least 19 genes involved in these Noonan syndrome spectrum disorders have been identified, the most common are PTPN11, SOS1, BRAF, HRAS, KRAS, NRAS, RAF1, RIT1, and SHOC2 [Grant et al., 2018].

Lymphatic dysplasia with a clinical lymphatic abnormality is one of the major features of Noonan syndrome spectrum disorders [van der Burgt, 2007]. Lymphatic vessels and lymph nodes make up the lymphatic system, which returns interstitial fluid to the venous system via the thoracic duct. When this system fails, the fluid is not returned to the blood system and accumulation of lymph can occur such as lymphedema, chylothorax, or ascites [Moore and Bertram, 2018]. Clinically relevant lymphatic abnormalities are a common finding in patients with NS, with an estimated lifetime prevalence of $20 \%$, although the exact number is still unknown[van der Burgt, 2007; Romano et al., 2010]. Lymphatic abnormalities in NS are not described in depth in most studies. Published studies about lymphatic abnormalities are limited to small cohorts, case series, and case reports. The most commonly described lymphatic abnormality is lymphedema, which usually resolves in early childhood when presented at birth; however, onset in later childhood and adulthood have also been described [Romano et al., 2010]. More severe complications include chylothorax and ascites. NS is also associated with ultrasound findings of lymphatic abnormalities in the prenatal phase, such as increased nuchal translucency (NT), pleural effusions, cystic hygroma, and distended jugular lymphatic sac [Haak et al., 2002; Bellini and Hennekam, 2012; Stuurman et al., 2018].

We performed a systematic review to gain more insight in the prevalence of lymphatic abnormalities in patients with genetically proven Noonan syndrome spectrum disorders including NS, NSML, NS-LAH, CS, and
CFCS, and to get more insight in a possible genotypelymphatic phenotype relationship. In addition, we looked at the clinical presentation and course of the lymphatic abnormality in different stages of life linked to specific genes. This is increasingly important because the activation of the RAS/MAPK pathway can be inhibited by RAS/ MAPK inhibitors. Recently, this led to the successful use of a MEK inhibitor in patients with NS and severe lymphatic abnormalities [Li et al., 2019; Dori et al., 2020].

\section{Methods}

\section{Search Strategy}

An electronic search in PubMed and EMBASE was conducted to obtain all publications on studies concerning Noonan syndrome spectrum disorders and lymphatic abnormalities. To identify relevant studies, selected medical subject heading ( $\mathrm{MeSH}$ ) terms and text words were used. MeSH terms used included "Noonan syndrome" or "Costello Syndrome" or "cardiofaciocutaneous Syndrome" or "Loose anagen hair syndrome" or "LEOPARD syndrome" or text words "Noonan" or "RASopathy*" or "Costello" or "LEOPARD" or "CFCS" or "cardiofaciocutaneous syndrome" or "cardio-facio-cutaneous syndrome" combined with MeSH terms "lymphatic abnormalities" or "lymphatic diseases" or "lymphatic system" or "chyle" or "lymphangiectasis" or "lymphedema" or "lymphocele" or "lymph" or "thoracic duct" or "nuchal translucency measurement" or "hydrops fetalis" or "chylothorax" or "pleural effusion" or text words "lymph*" or "chyle" or "thoracic duct" or "nuchal fold" or "nuchal translucency" or "hydrops fetal*" or "chylothorax" or "pleural effusion".

\section{Inclusion}

Studies of patients with genetically confirmed NS, NSML and NS-LAH, CFCS, and CS and in which the lymphatic abnormality was described, published before September 1, 2020 were included. The classification of gene-disease association by Grant et al. [2018] was used if possible. As the number of publications about a lymphatic phenotype in Noonan syndrome spectrum diseases was felt to be limited, no study designs were excluded. Excluded were studies published before the year 2000 (due to the absence of gene studies in that period), studies published in a language other than English, animal studies, letters to editors, books, conference abstracts, and studies with low quality, as explained by the criteria below. References of the eligible articles were searched to identify studies that did not appear in our search. To assess whether the inclusion criteria were met, all articles were screened by title and abstract by 3 investigators (J.S., J.D., W.K.). Full-text screening was performed by 2 investigators (J.S., J.D.). Search results were collected and deduplicated in Endnote and then exported into Rayyan software (https://rayyan.qcri.org) for ease of management. Conflicts were discussed to reach consensus.

\section{Quality Assessment}

Methodological quality of included articles was assessed according to the Joanna Briggs checklists. The Joanna Briggs Institute (JBI) developed critical appraisal tools for cohort studies, case reports and case series, approved by the JBI Scientific Com- 


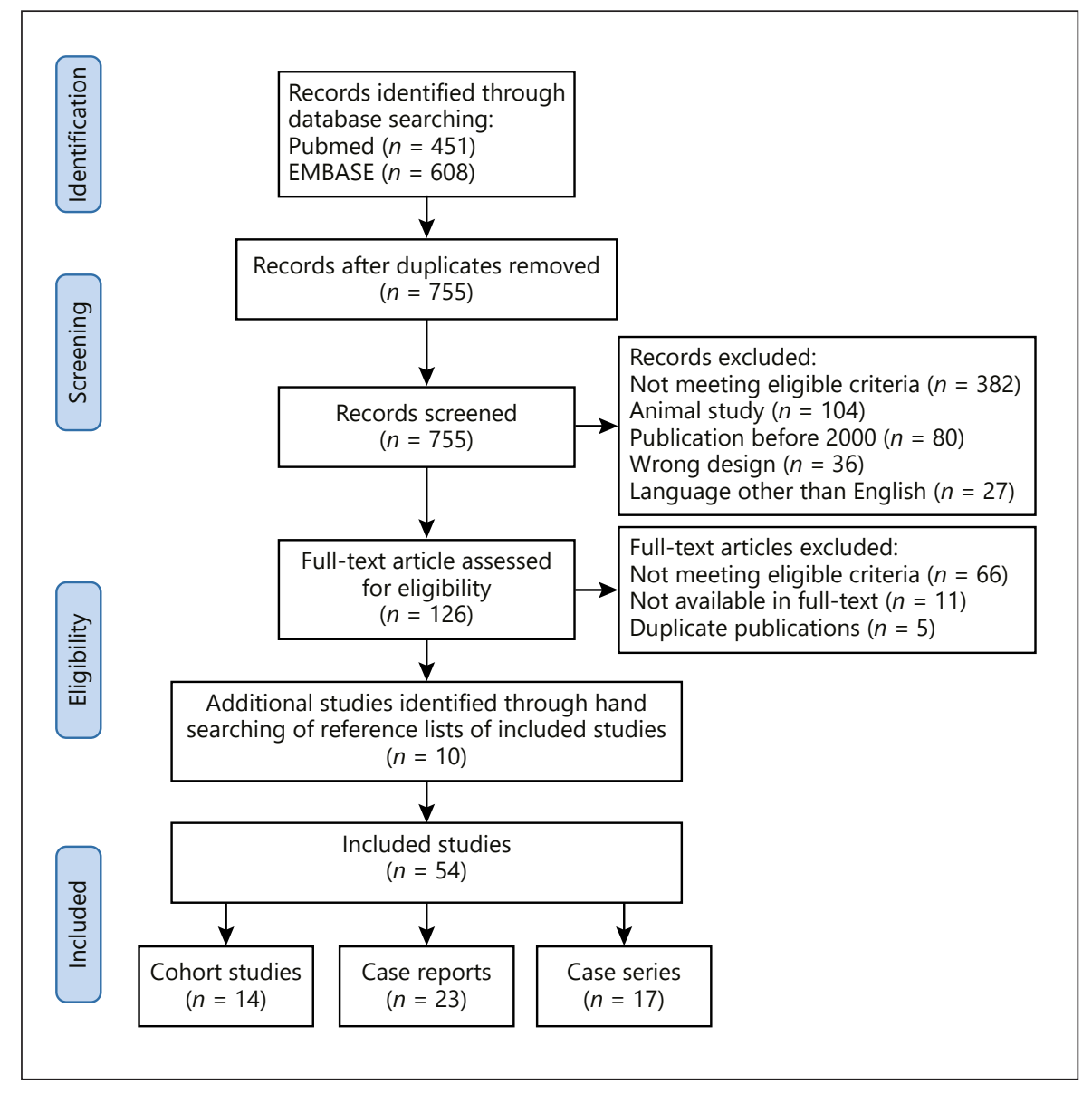

Fig. 1. Flow diagram of included studies.

mittee [Moola et al., 2020]. Questions not applicable were removed from the checklist for that particular article. Quality of the studies was rated as high quality if at least $75 \%$ of the remaining questions could be answered with "yes". If the answer could be yes on $50 \%$ or higher, quality was rated as moderate [Drovandi et al., 2018]. Quality assessment was performed by 2 investigators (J.S., J.D.)

\section{Data Extraction and Descriptive Analysis}

Results from cohort studies and case series were extracted from text and tables. Results from case reports were extracted from texts and photo captions. Data extraction was performed by one author (J.S.) in co-operation with a second author (J.D.). The cohort studies were used for describing the prevalence of lymphatic abnormalities. The number of patients of the cohorts with more than 10 patients were compared and, when possible, summarized to create a greater perspective. When more than $10 \mathrm{pa}-$ tients per gene were included, a statistical analysis between genes was performed to evaluate if there was a statistically meaningful difference in prevalence of lymphatic abnormalities. Statistical significance was analyzed using Fisher's exact test, with a significance threshold of $p \leq 0.05$. The clinical phenotype in different stages of life, causing gene, and severity of symptoms were analyzed using the cumulative data of case reports and case series whenever possible.

Lymphatic Abnormalities in Rasopathies

\section{Results}

\section{Literature Search}

The literature searches through PubMed and EMBASE resulted in 451, respectively 608 hits. After duplicates were removed, a total of 755 articles were screened. Based on title and abstract, 629 articles had to be excluded. Of 126 articles the full-text copy was acquired. A total of 77 studies were excluded because they did not meet the eligibility criteria or were not available in full text. Searching the references resulted in 10 additional publications. In total, 54 studies were included: 14 cohort studies, 23 case reports, and 17 case series. Details are summarized in Figure 1.

\section{Study Quality}

Quality assessment of the 54 studies was performed using the Joanna Briggs criteria [Moola et al., 2020]. Of the cohort studies, 1 was rated as moderate quality, 13 as high quality. Of the case reports, 22 were rated as high quality, 1 was rated as moderate quality. All 17 case series were rated as high quality. No studies were rated as low quality. 
Table 1. Prenatal ultrasound findings suggestive for lymphatic diseases in NS caused by pathologic variants in specific genes

\begin{tabular}{lll}
\hline & Gene & Incidence \\
\hline Increased NT & PTPN11 & $3 / 41(7 \%)$ \\
& SOS1 & $2 / 6(33 \%)$ \\
& RAF1 & $1 / 2(50 \%)$ \\
& KRAS & $2 / 2(100 \%)$ \\
& RIT1 & $14 / 37(38 \%)^{*}$ \\
\hline Pleural effusions & PTPN11 & $11 / 71(15 \%)$ \\
& SOS1 & $1 / 14(7 \%)$ \\
& RIT1 & $12 / 41(29 \%)$ \\
\hline Cystic hygroma & PTPN11 & $23 / 41(56 \%)$ \\
& SOS1 & $2 / 6(33 \%)$ \\
& RAF1 & $1 / 2(50 \%)$ \\
& BRAF & $1 / 1(100 \%)$ \\
& NRAS & $1 / 1(100 \%)$ \\
& RIT1 & $1 / 37(3 \%)^{*}$ \\
\hline Hydrops fetalis & PTPN11 & $7 / 41(17 \%)$ \\
& SOS1 & $1 / 6(17 \%)$ \\
\hline Pericardial effusions & RIT1 & $9 / 41(22 \%)$ \\
\hline Ascites & PTPN11 & $1 / 41(2 \%)$ \\
\hline \multirow{2}{*}{ * 01 versus PTPN11 } & $2 / 41(5 \%)$ \\
\hline & PTPN11 & \\
\hline
\end{tabular}

\section{Prevalence of Lymphatic Diseases}

Noonan Syndrome

Data about prenatal and postnatal lymphatic abnormalities in patients with NS could be extracted from 11 cohort studies. Combining these cohorts resulted in 296 patients with NS [Zenker et al., 2004; Schubbert et al., 2006; Shaw et al., 2007; Ko et al., 2008; Lee et al., 2009; Baldassare et al., 2011; Digilio et al., 2011; Smpokou et al., 2012; Hakami et al., 2016; Kouz et al., 2016; Yaoita et al., 2016]. Germline pathogenic variants in patients with NS were found in PTPN11 $(n=182)$, RIT1 $(n=47)$, SOS1 $(n=40)$, BRAF $(n=4), R A F 1(n=11)$, KRAS $(n=9)$, NRAS $(n=2)$, and $C B L(n=1)$. One cohort reported a lifetime prevalence of lymphatic abnormalities in patients with NS caused by pathogenic variants in PTPN11 and SOS1 of 55\% (11/20) and 25\% (2/8), respectively [Digilio et al., 2011] The other cohorts were subdivided into prenatal and postnatal phase.

In the prenatal phase, the prevalence differed according to the ultrasound findings of lymphatic abnormality and the causative gene. The prevalence of increased NT differed from $7 \%$ in patients with PTPN11 to $38 \%$ in patients with RIT1 (PTPN11 vs. RIT1: $p<0.01$ ); the prevalence of pleural effusions differed from $7 \%$ in patients
Table 2. Postnatal lymphatic diseases in NS caused by pathologic variants in specific genes

\begin{tabular}{|c|c|c|}
\hline Lymphatic disease & Gene & Prevalence \\
\hline \multirow{3}{*}{ Lymphedema } & PTPN11 & $17 / 103(16 \%)^{*}$ \\
\hline & SOS1 & $8 / 18(44 \%)$ \\
\hline & RIT1 & $7 / 46(15 \%)^{*}$ \\
\hline Neonatal lymphedema & RIT1 & $7 / 46(15 \%)$ \\
\hline Acquired lymphedema & RIT1 & $5 / 46(10 \%)$ \\
\hline Neonatal chylothorax & RIT1 & $3 / 46(6 \%)$ \\
\hline Acquired chylothorax & RIT1 & $2 / 46(4 \%)$ \\
\hline $\begin{array}{l}\text { Ascites/protein-losing } \\
\text { enteropathy }\end{array}$ & RIT1 & $2 / 46(4 \%)$ \\
\hline
\end{tabular}

with SOS1 to $29 \%$ in patients with RIT1 (not statistically significant between genes); the prevalence of cystic hygroma differed form $3 \%$ in patients with RIT1 to $56 \%$ in patients with PTPN11 (RIT1 vs. PTPN11: $p<0.01$ ), and the prevalence of hydrops fetalis differed from $17.1 \%$ in patients with PTPN11 to $22 \%$ in patients with RIT1 (not statistically significant between genes). The results are summarized in Table 1 [Zenker et al., 2004; Schubbert et al., 2006; Shaw et al., 2007; Ko et al., 2008; Lee et al., 2009; Baldassare et al., 2011; Smpokou et al., 2012; Hakami et al., 2016; Kouz et al., 2016; Yaoita et al., 2016].

In the postnatal phase, the prevalence differed according to the lymphatic abnormality and causative gene. The prevalence of lymphedema differed from $15 \%$ in patients with RIT1, $16 \%$ in patients with PTPN11, to $44 \%$ in patients with SOS1 (RIT1 vs. SOS1: $p<0.01$; PTPN11 vs. SOS1; $p<0.01$ ); the prevalence of acquired chylothorax was $4 \%$ in patients with RIT1, and the prevalence of protein losing enteropathy/ascites was also $4 \%$ in patients with RIT1. The results are summarized in Table 2 [Zenker et al., 2004; Schubbert et al., 2006; Shaw et al., 2007; Ko et al., 2008; Lee et al., 2009; Baldassare et al., 2011; Smpokou et al., 2012; Hakami et al., 2016; Kouz et al., 2016; Yaoita et al., 2016].

Twelve NSML patients due to pathogenic variants in PTPN11 and 2 NS-LAH patients due to pathogenic variants in SHOC2 were reported [Baldassare et al., 2011; Digilio et al., 2011]. Three patients with NSML were found to have prenatal or neonatal lymphatic abnormalities, not specified in detail. Digilio et al. [2011] reported no lymphatic diseases in NS-LAH syndrome.

Cardiofaciocutaneous Syndrome

Seventy-eight patients with CFCS were reported with pathogenic variants of BRAF $(n=73), \operatorname{MEK2}(n=1)$,
Mol Syndromol 2022;13:1-11 DOI: $10.1159 / 000517605$
Sleutjes/Kleimeier/Leenders/Klein/ Draaisma 
Fig. 2. Period of onset and follow-up of increased nuchal translucency in patients with Noonan syndrome.

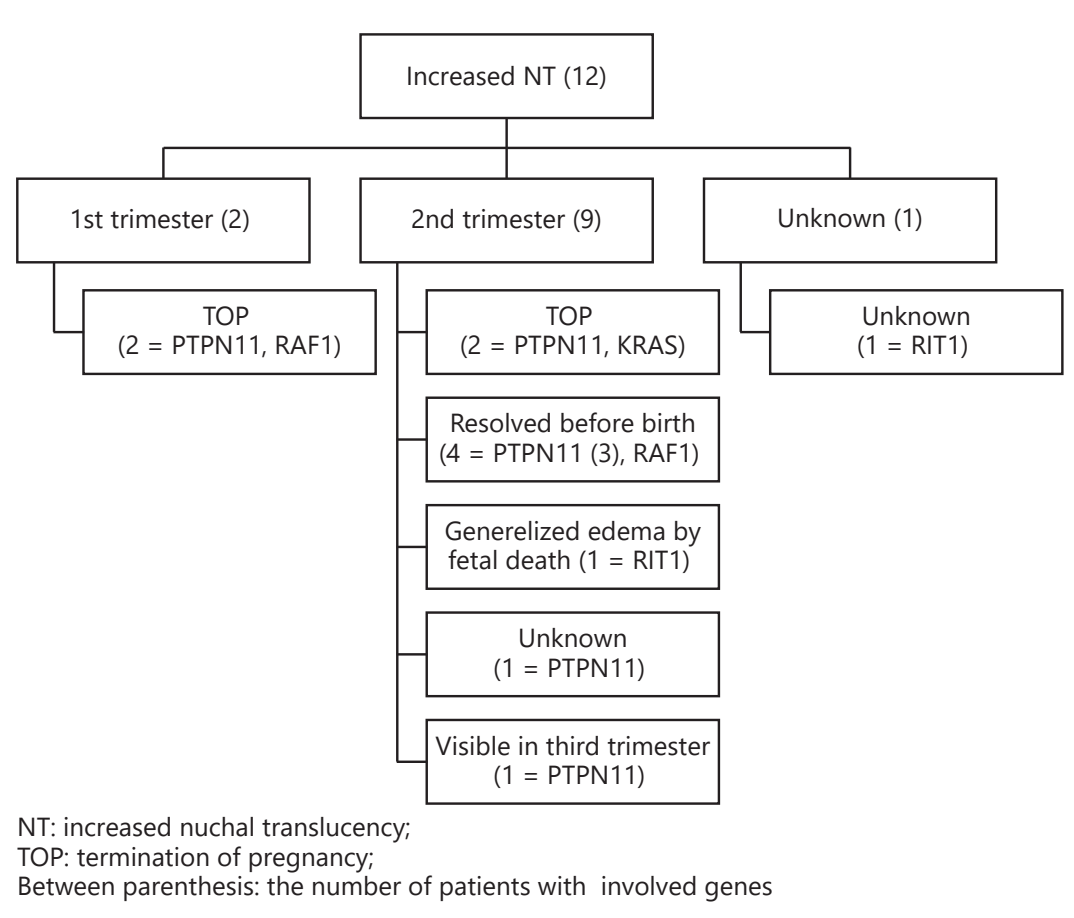

KRAS $(n=1)$, MAP2K1 $(n=1), P T P N 11(n=1)$, and RAF1 $(n=1)$. [Armour and Allanson, 2008; Ko et al., 2008; Baldassare et al., 2011, Digilio et al., 2011; Kouz et al., 2016]. The diagnosis of the last 2 cases can be disputed according to Grant et al. [2018]. In the prenatal phase, the prevalence of increased NT was $13 \%$ in patients with a pathogenic $B R A F$ variant [Templin et al., 2016]. The lifetime prevalence in CFCS caused by a pathogenic BRAF variant was 50\% (2/4) [Digilio et al., 2011]. Another study reported pleural effusions in 3\% (1/39), without mentioning a specific mutation [Armour and Allanson, 2008]. In the postnatal phase, lymphedema was reported sporadically.

\section{Costello Syndrome}

Eight patients with CS were reported, showing that prenatal cystic hygroma and prenatal and postnatal hydrops fetalis do occur [Baldassarre et al., 2011; Digilio et al., 2011; Templin et al., 2016]. Digilio et al. [2011] described a lifetime prevalence of lymphatic diseases of $50 \%$ $(2 / 4)$.

\section{Clinical Presentation in Different Stages of Life}

In total, 78 cases were described in 23 case reports and 17 case series. NS was reported in 50 cases and NS-LAH in 2 cases. In NS pathogenic variants were found in PTPN11 $(n=28)$, RAF1 $(n=6), C B L(n=4), \operatorname{SOS1}(n=$
2), KRAS ( $n=1), \operatorname{RIT1}(n=6)$, SOS2 $(n=1)$ and ARAF $(n=2)$. In 2 patients with NS-LAH pathogenic variants were found in SHOC2. In CFCS pathogenic variants were found in BRAF $(n=5), \operatorname{KRAS}(n=4)$ and MAP2K2 $(n=$ 2). In 15 patients with CS, HRAS pathogenic variants were found.

\section{Noonan Syndrome}

Prenatal Findings: abnormal ultrasound findings included: increased NT, cystic hygroma, distended jugular lymphatic sacs, effusions, and ascites. Details about period of onset, causing gene variant, and follow-up of prenatal ultrasound findings in NS are summarized for NT and effusions in Figure 2 and Figure 3 [Yoshida et al., 2004; Schlüter et al., 2005; Bekker et al., 2007; Fabretto et al., 2010; Gonzalez-Huerta et al., 2010; Houweling et al., 2010; Bakker et al., 2011; Kayiran et al, 2013; Bülow et al., 2015; Ford and Trotter, 2015; Kneitel et al., 2015; Joyce et al., 2016; Milosavljevic et al., 2016; Thompson et al., 2017]. Two case reports described NS-LAH with increased NT, of which 1 case also showed hydrops fetalis, ascites, and skin edema caused by a pathogenic variant in SHOC2 [Gargano et al., 2014; Zmolikova et al., 2014].

Postnatal Findings: the described postnatal lymphatic abnormalities included: lymphedema, chylothorax, chylopericardium, chylous ascites, and protein losing enter- 


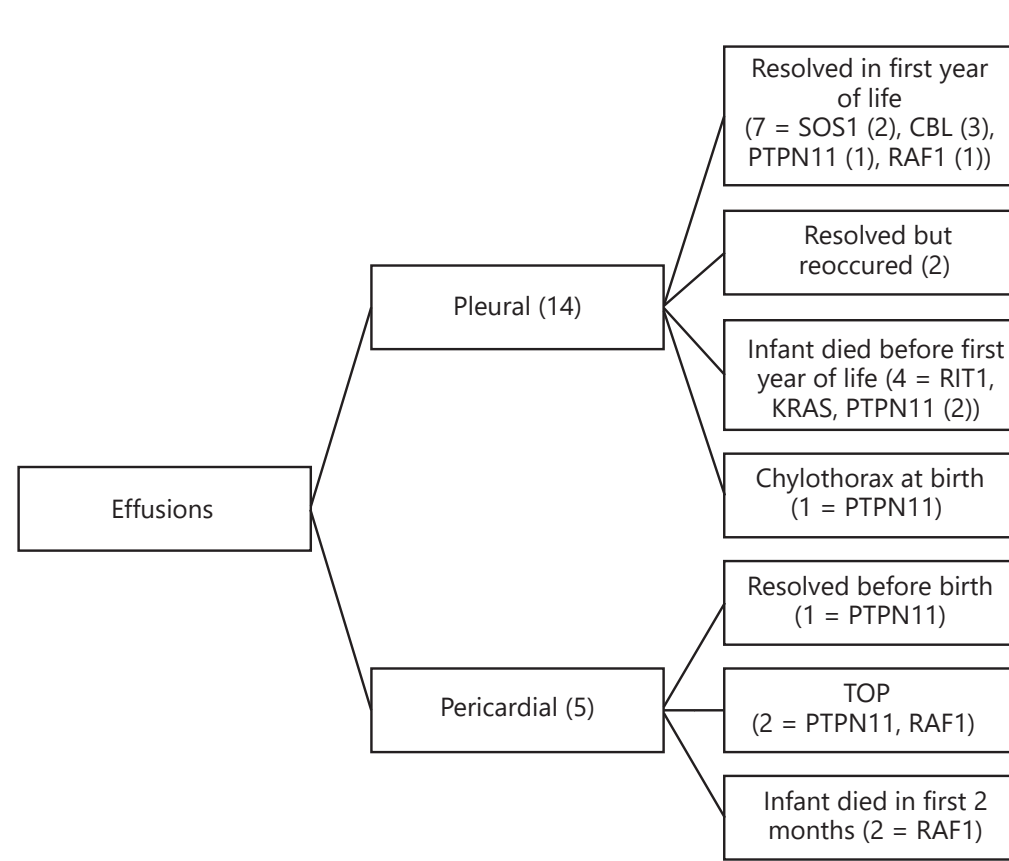

DOL: day of life; TOP: termination of pregnancy;

Between parenthesis: the number of patients with involved genes

Fig. 3. Date of onset, follow-up, and gene defects of effusions developed during pregnancy in Noonan syndrome.

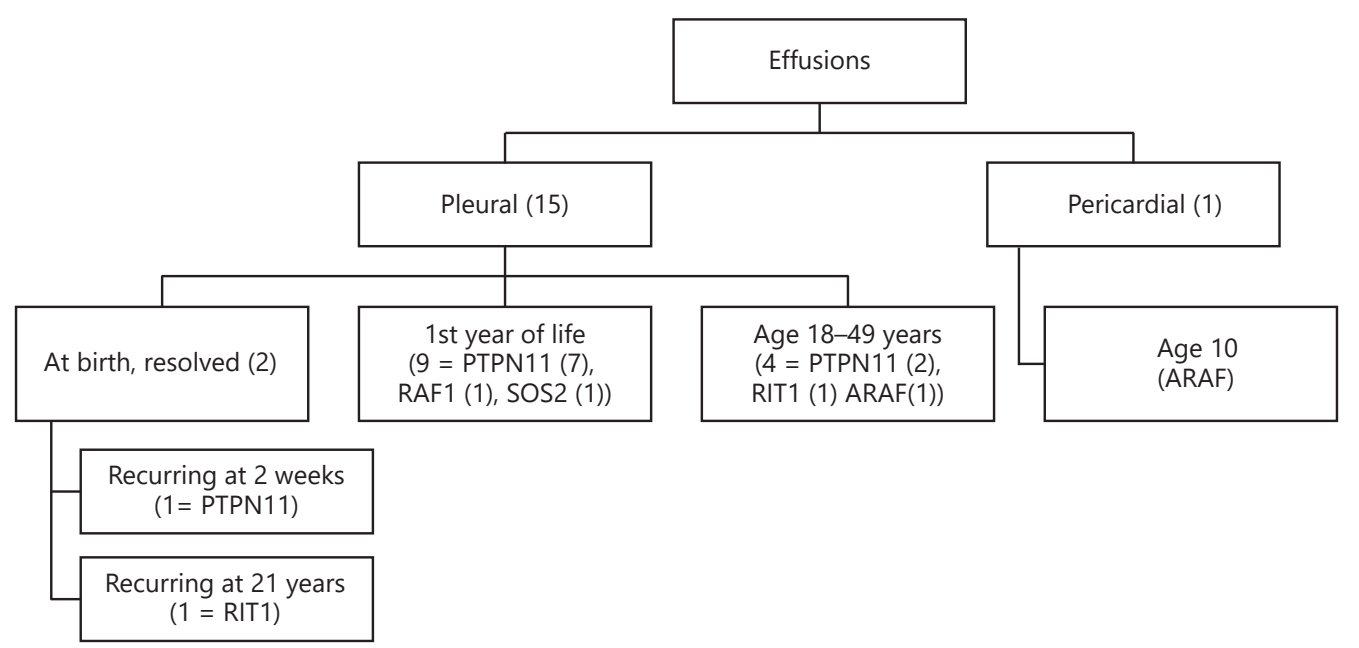

Pleural: pleural effusions; Pericardial: pericardial effusions

Between parenthesis: the number of patients with involved genes

Fig. 4. Date of onset, follow-up, and gene defects of effusions developed postnatally in Noonan syndrome. 
opathy. Chylous effusions, including pericardial and pleural effusions, were described in 16 cases with NS. The date of onset of chylous pleural effusions ranged from birth to 49 years, with a median of 1.9 months. Also the development of a chylothorax after cardiac surgery in a 49-year-old male was described [Joyce et al., 2016; Milosavljevic et al., 2016]. Details about age of onset, followup, and disease-causing genes are summarized in Figure 4 [Mathur et al., 2014; Joyce et al., 2016; Ebrahimi-Fakhari et al., 2017; Biko et al., 2019; Ding et al., 2019; Li et al., 2019].

Lower limb lymphedema and genital lymphedema are the 2 most reported types of lymphedema that developed after birth. Fifteen cases of lower limb lymphedema and 8 cases of genital lymphedema were described in NS. The age of onset of lower limb lymphedema ranged from 3 to 55 years, with a median of 10 years [Hanson et al., 2014; Bülow et al., 2015; Joyce et al., 2016; Koenighofer et al., 2016; Milosavljevic et al., 2016; Ding et al., 2019; Li et al., 2019; Wang et al., 2020]. Onset of genital lymphedema ranged from 3 to 63 years, with a median of 8 years. All cases with genital lymphedema were also found to have lower limb lymphedema. In 8 cases, genital edema was found, whereas genital lymph blisters were found in 3 cases. One report described genital lymphatic dysplasia in the dermis of the vulva as the only symptom [Lee et al., 2010]. In NS, protein-losing enteropathy was found in 3 cases; intestinal lymphangiectasia was described separately in 1 case [Joyce et al., 2016; Wang et al., 2020].

\section{Cardiofaciocutaneous Syndrome}

Prenatal Findings: increased NT was described once, caused by a pathogenic BRAF variant [Witters et al., 2008]. Cystic hygroma was found in 4 cases, caused by pathogenic variants in BRAF $(n=3)$ and MAP2K1 $(n=1)$ [Witters et al., 2008; Terry et al., 2014; Mucciolo 2016; Biard et al., 2019]. No pericardial and pleural effusions were described; 1 case showed ascites [Terry et al., 2014].

Postnatal Findings: effusions were described in 4 cases, of which 1 was pericardial [Akahoshi et al., 2020], caused by a pathogenic KRAS variant, and 3 pleural [Morcaldi et al., 2015; Joyce et al., 2016], caused by pathogenic variants in KRAS $(n=2)$ and BRAF $(n=1)$. Five cases were described with lower limb edema and 3 also with genital involvement [Morcaldi et al., 2015; Joyce et al., 2016; Akahoshi et al., 2020]. Protein-losing enteropathy was described in 1 case, intestinal lymphangiectasia in another [Joyce et al. [2016]. One case series reported a family with CFCS due to a pathogenic MEK2 variant without lymphatic involvement [Rauen et al., 2010].

Lymphatic Abnormalities in Rasopathies
Costello Syndrome

Prenatal Findings: increased NT was found in 3 cases, prenatal pericardial effusions were described in 1 case, pleural effusions were described in 5 cases [Lo et al., 2008; Kuniba et al., 2009; Lin et al., 2009; Bertola et al., 2017]. One report described only scalp and truncal edema, another only edema in the second trimester [Sinico et al., 2011; Bend et al., 2019].

Postnatal Findings: pleural effusions were described in 1 case [Lo et al., 2008]. Also 1 case with lower limb edema was described [Bertola et al., 2017].

\section{Discussion}

This systematic review gives an overview of lymphatic abnormalities in patients with genetically proven Noonan syndrome spectrum disorder. For this reason, we excluded NF1 and Legius syndrome [Villani et al., 2017; Grant et al., 2018]. The first research question was aimed at the prevalence of lymphatic diseases in the different Noonan syndrome spectrum disorders and if a genotype-lymphatic phenotype relation could be found; the second research question concerned the clinical presentation and course of lymphatic abnormalities in different phases of life. Prenatal ultrasound in NS has a prevalence of $25 \%$ for increased NT, $19 \%$ for pleural effusions, and 33\% for cystic hygroma. Additional literature shows a cohort of 25 disease-causing variant positive fetuses, all tested for 9 different genes, including NS, CFC syndrome and CS, in which $64 \%$ had increased NT, $44 \%$ pleural effusions, and $43 \%$ jugular lymphatic sacs [Stuurman et al., 2018]. This article was not included in the systematic review because there was no differentiation between the 3 syndromes. The different cohort studies did not all test for the same genes, which can be an explanation for the different outcomes. Digilio et al. [2011] reported a prevalence of prenatal/neonatal lymphatic abnormalities in NS due to pathogenic variants in PTPN11 and SOS1 of 55\% and $25 \%$, respectively. Additional literature states that lymphatic abnormalities are observed in $20 \%$ of the patients with NS [van der Burgt, 2007]. However, this number is based on the clinical diagnosis or a pathogenic variant in PTPN11. Two cohorts reported postnatal lymphatic abnormalities caused by pathogenic variants in RIT1, resulting in a combined prevalence of 33\% [Kouz et al., 2016; Yaoita et al., 2016]. Two other cohorts reported postnatal lymphedema in NS caused by a SOS1 mutation, resulting in a combined prevalence of $44 \%$ [Ko et al., 2008; Smpokou et al., 2012]. The numbers and descrip-

Mol Syndromol 2022;13:1-11

DOI: $10.1159 / 000517605$ 
tions of lymphatic abnormalities in genes other than PTPN11 suggest that the prevalence of lymphatic diseases in NS may be more than $20 \%$, combining all the diseasecausing genes currently known. This can be augmented by a recently published study on NS patients due to a disease-causing SOS2 variant, in which lymphatic abnormalities affected more than half of the patients [Lissewski et al., 2021].

Our second research question concerned the presentation of lymphatic abnormalities in different stages of life. According to Menashe et al. [2002] ultrasound findings of prenatal lymphatic abnormalities present after the first trimester. However, as was shown in this systematic review, the initial presentation of NT was in the first trimester in $17 \%$ and in $75 \%$ in the second trimester. In some cases with presentation in the second trimester, it was described that the increased NT resolved before the third trimester, in one case the increased NT was still visible in the third trimester. These findings are in contrast with additional literature, which shows a cohort of 15 fetuses with increased NT visible in the first trimester, still visible in the second and third trimester in 89\% [Vigneswaran et al., 2018].

Cystic hygroma and distended jugular lymphatic sacs were also described. Interestingly, $100 \%$ of the cases with distended jugular lymphatic sacs also presented with increased NT, a correlation earlier suggested in literature [Haak et al., 2002]. Prenatal pleural effusions in NS were diagnosed in the second or third trimester. Pleural effusions presenting in the second trimester were still visible in the third trimester or at birth in $100 \%$, a finding also earlier described in literature [Vigneswaran et al., 2018].

Age of presentation of postnatal chylous pleural effusions in NS was described in 15 cases and ranged from birth to 49 years. In one case, effusions were present at birth and reoccurred at age 21 [Joyce et al., 2016].

No other cases describing reappearance were found. Another interesting finding is the development of chylothorax after cardiac surgery in a 49-year-old male with NS. Postoperative chylothorax is described in literature before [Joyce et al., 2016]. A case of a 28-year-old male who developed pleural chylous effusions and worsening of lower lymphedema was reported [Bottner et al., 2005]. Likewise, in a large cohort of 778 patients with NS who underwent cardiac surgery, postoperative chylothorax developed in $2 \%$. Patients with NS were found to be associated with increased odds of chylothorax [Kriz et al., 2019].

The presentation of lymphedema in NS can vary over time from fetal to adult age. The age of initial presentation of lower limb edema described in the included cases ranged from 3 to 55 years and was sometimes complicated by genital involvement. One of them also showed protein losing enteropathy [Hasegawa et al., 2009]. Previous research suggested that the lymphatic phenotype may be present in various ways, including variation in timing of appearance and resolution of edema at different ages. Prenatal onset of lymphatic abnormalities might result in a more severe phenotype, whereas postnatal presentation may result in a milder phenotype [Haque et al., 2012]. We looked at the most common postnatal presentations. Based on this literature review, we can agree with Haque et al. [2012] that later onset of lymphatic abnormalities in NS might result in a milder phenotype.

The prevalence of NSML and NS-LAH is not precisely known, with an estimated amount of 1/100,000 and 100 reports, respectively [Tajan et al., 2018] Two cohorts containing NS-ML reported a lifetime prevalence of lymphatic abnormalities in 25\% [Digilio et al., 2011; Hakami et al., 2016]. No case reports or series containing NSML were found. Two reports and one cohort described NSLAH due to SHOC2-mutations [Digilio et al., 2011; Gargano et al., 2014; Zmolikova et al., 2014]. Gargano et al. [2014] described a more severe phenotype including, hydrops fetalis, pleural effusions, and generalized edema resulting in death. Since these are very rare conditions, there is not much literature available about the presentation and lymphatic abnormalities. Therefore, it can only be concluded that lymphatic abnormalities may present in patients with NS-LAH or NSML.

In the prenatal phase, the prevalence of increased NT in CFCS is about $13 \%$ in patients with a pathogenic BRAF variant. Cystic hygroma is also described. However, no pleural effusions are described. In the postnatal phase, lymphedema in patients with a pathogenic $K R A S$ variant is sporadically described, often accompanied by pleural effusions. This may suggest that pathogenic KRAS variants associated with CFCS are more likely to result in postnatal effusions than KRAS variants associated with NS [Morcaldi et al., 2015; Joyce et al., 2016]

Only few patients with CS could be included, showing that prenatal cystic hygroma and prenatal and postnatal hydrops fetalis do occur. Postnatal lymphedema does occur.

\section{Study Limits}

There are several limitations of this review. Publication bias can play a role. In the cohorts, different inclusion criteria and methods were used; therefore differences were inevitable. Furthermore, the case reports and case 
series we included did not present their findings in the same way. Another limitation might be the amount of currently published data. Only limited studies are available about postnatal lymphatic abnormalities in Noonan syndrome spectrum disorders with a proven germline pathogenic variant. As the numbers are small, the risk of over- or underestimation should be taken into account.

\section{Conclusion}

Noonan syndrome spectrum disorders, NS in particular, are associated with lymphatic diseases. Combining the available published literature about genetically proven Noonan syndrome spectrum disorders, it appears likely that the lifetime prevalence of these anomalies in NS is higher than the $20 \%$ that was generally accepted so far, and that the presentation and course of lymphatic abnormalities may be severe and even lethal. Further cohort studies including all gene variants currently known for Noonan syndrome spectrum disorders should address this theory. This is increasingly important because the activation of the RAS/MAPK pathway can be inhibited by
RAS/MAPK inhibitors. Recently, this led to the successful use of a MEK inhibitor in patients with NS and severe lymphatic abnormalities.

\section{Conflict of Interest Statement}

The authors have no conflicts of interest to declare. Several author(s) of this publication are members of the European Reference Network for Developmental Anomalies and Intellectual Disability (ERN-ITHACA).

\section{Funding Sources}

The authors received no funding sources.

\section{Author Contributions}

Julia Sleutjes: data analysis and interpretation, drafting of the manuscript. Lotte Kleimeier: drafting of the manuscript. Erika Leenders: critical review of the manuscript. Willemijn Klein: data analysis and interpretation, drafting of the manuscript. Jos Draaisma: data analysis and interpretation, critical review of the manuscript. All authors contributed to the conceptualisation and design of the study and approved the final version of the manuscript.

\section{References}

Akahoshi S, Hirano A, Nagamine H, Miura M. Cardiofaciocutaneous syndrome with KRAS gene mutation presenting as chylopericardium. Am J Med Genet A. 2020;182(3):532-5.

Armour CM, Allanson JE. Further delineation of cardio-facio-cutaneous syndrome: clinical features of 38 individuals with proven mutations. J Med Genet. 2008;45(4):249-54.

Bakker M, Pajkrt E, Mathijssen IB, Bilardo CM. Targeted ultrasound examination and DNA testing for Noonan syndrome, in fetuses with increased nuchal translucency and normal karyotype. Prenat Diagn. 2011;31(9):833-40.

Baldassarre G, Mussa A, Dotta A, Banaudi E, Forzano S, Marinosci A, et al. Prenatal features of Noonan syndrome: prevalence and prognostic value. Prenat Diagn. 2011;31(10):949-54.

Bekker MN, Go AT, van Vugt JM. Persistence of nuchal edema and distended jugular lymphatic sacs in Noonan syndrome. Fetal Diagn Ther. 2007;22(4):245-8.

Bellini C, Hennekam RC. Non-immune hydrops fetalis: a short review of etiology and pathophysiology. Am J Med Genet A. 2012;158A(3): 597-605.

Bend EG, Louie RJ, Stevenson RE. Fetal edema, not overgrowth, is associated with neonatal lethal Costello syndrome due to the HRAS p.Gly12Val mutation. Clin Dysmorphol. 2019;28(2):71-3.

Lymphatic Abnormalities in Rasopathies
Bertola D, Buscarilli M, Stabley DL, Baker L, Doyle D, Bartholomew DW, et al. Phenotypic spectrum of Costello syndrome individuals harboring the rare HRAS mutation $p$. Gly13Asp. Am J Med Genet A. 2017;173(5): 1309-18.

Biard JM, Steenhaut P, Bernard P, Race V, Sznajer Y. Antenatal diagnosis of cardio-facio-cutaneous syndrome: Prenatal characteristics and contribution of fetal facial dysmorphic signs in utero. About a case and review of literature. Eur J Obstet Gynecol Reprod Biol. 2019;240: $232-41$.

Biko DM, Reisen B, Otero HJ, Ravishankar C, Victoria T, Glatz AC, et al. Imaging of central lymphatic abnormalities in Noonan syndrome. Pediatr Radiol. 2019;49(5):586-92.

Bottner F, Sandmann C, Semik M, Ramm O, Winkelmann W, Liljenqvist U. Chylothorax after surgery for thoracic deformity in Noonan syndrome. Orthopedics. 2005;28(1):71-3.

Bülow L, Lissewski C, Bressel R, Rauch A, Stark Z, Zenker M, et al. Hydrops, fetal pleural effusions and chylothorax in three patients with CBL mutations. Am J Med Genet A. 2015; $167 \mathrm{~A}(2): 394-9$

Digilio MC, Lepri F, Baban A, Dentici ML, Versacci P, Capolino R, et al. RASopathies: Clinical Diagnosis in the First Year of Life. Mol Syndromol. 2011;1(6):282-9.
Ding Y, Hu XY, Song YN, Cao BY, Liang XJ, Li $\mathrm{HD}$, et al. A report on a girl of Noonan syndrome 9 presenting with bilateral lower limbs lymphedema. Chin Med J (Engl). 2019; 132(4):480-2.

Dori Y, Smith C, Pinto E, Snyder K, March ME, Hakonarson H, et al. Severe Lymphatic Disorder Resolved With MEK Inhibition in a Patient With Noonan Syndrome and SOS1 Mutation. Pediatrics. 2020; 146(6): e20200167.

Drovandi A, Robertson K, Tucker M, Robinson N, Perks S, Kairuz T. A systematic review of clinical pharmacist interventions in paediatric hospital patients. Eur J Pediatr. 2018; 177(8):1139-48.

Ebrahimi-Fakhari D, Freiman E, Wojcik MH, Krone K, Casey A, Winn AS, et al. Congenital Chylothorax as the Initial Presentation of PTPN11-Associated Noonan Syndrome. J Pediatr. 2017;185:248-248e1.

Fabretto A, Kutsche K, Harmsen MB, Demarini S, Gasparini P, Fertz MC, et al. Two cases of Noonan syndrome with severe respiratory and gastroenteral involvement and the SOS1 mutation F623I. Eur J Med Genet. 2010;53(5): $322-4$.

Ford JJ, Trotter CW. Noonan Syndrome Complicated by Primary Pulmonary Lymphangiectasia. Neonatal Netw. 2015;34(2):117-25. 
Gargano G, Guidotti I, Balestri E, Vagnarelli F, Rosato S, Comitini G, et al. Hydrops fetalis in a preterm newborn heterozygous for the c. $4 \mathrm{~A}>\mathrm{G}$ SHOC2 mutation. Am J Med Genet A. $2014 ; 164 \mathrm{~A}(4): 1015-20$.

Gonzalez-Huerta NC, Valdes-Miranda JM, Perez-Cabrera A, Pacheco-Cuellar G, GonzalezHuerta LM, Cuevas-Covarrubias SA. Noonan syndrome: prenatal diagnosis in a woman carrying a PTPN11 gene mutation. J Matern Fetal Neonatal Med. 2010;23(7):688-91.

Grant AR, Cushman BJ, Cavé H, Dillon MW, Gelb BD, Gripp KW, et al. Assessing the genedisease association of 19 genes with the RASopathies using the ClinGen gene curation framework. Hum Mutat. 2018;39(11):148593.

Haak MC, Bartelings MM, Jackson DG, Webb S, van Vugt JM, Gittenberger-de Groot AC. Increased nuchal translucency is associated with jugular lymphatic distension. Hum Reprod. 2002;17(4):1086-92.

Hakami F, Dillon MW, Lebo M, Mason-Suares H. Retrospective study of prenatal ultrasound findings in newborns with a Noonan spectrum disorder. Prenat Diagn. 2016;36(5): $418-23$.

Hanson HL, Wilson MJ, Short JP, Chioza BA, Crosby AH, Nash RM, et al. Germline CBL mutation associated with a noonan-like syndrome with primary lymphedema and teratoma associated with acquired uniparental isodisomy of chromosome 11q23. Am J Med Genet A. 2014;164A(4):1003-9.

Haque MA, Sharmin LS, Amin Z, Ekram AS. Noonan Syndrome Presenting as Lymphoedema Precox. J Medicine. 2012;13(2):24345.

Hasegawa K, Nagaoka Y, Maruyama H, Aya K, Tanaka H, Morishima T. Late-onset Lymphedema and Protein-losing Enteropathy with Noonan Syndrome. Clin Pediatr Endocrinol. 2009;18(3):87-93.

Houweling AC, de Mooij YM, van der Burgt I, Yntema HG, Lachmeijer AM, Go AT. Prenatal detection of Noonan syndrome by mutation analysis of the PTPN11 and the KRAS genes. Prenat Diagn. 2010;30(3):284-6.

Joyce S, Gordon K, Brice G, Ostergaard P, Nagaraja $\mathrm{R}$, Short J, et al. The lymphatic phenotype in Noonan and Cardiofaciocutaneous syndrome. Eur J Hum Genet. 2016;24(5): 690-6.

Kayiran PGT, Gürsoy T, Gürakan B. Non immune hydrops fetalis: Two premature infants with favorable outcome. Marmara Med J. 2013;26(3):168-70.

Kneitel AW, Norby A, Vettraino I, Treadwell MC. A Novel Mutation on RAF1 in Association with Fetal Findings Suggestive of Noonan Syndrome. Fetal Pediatr Pathol. 2015;34(6): 361-4.

Ko JM, Kim JM, Kim GH, Yoo HW. PTPN11, SOS1, KRAS, and RAF1 gene analysis, and genotype-phenotype correlation in Korean patients with Noonan syndrome. J Hum Genet. 2008;53(11-12):999-1006.
Koenighofer M, Hung CY, McCauley JL, Dallman J, Back EJ, Mihalek I, et al. Mutations in RIT1 cause Noonan syndrome - additional functional evidence and expanding the clinical phenotype. Clin Genet. 2016;89(3):359-66.

Kouz K, Lissewski C, Spranger S, Mitter D, Riess A, Lopez-Gonzalez V, et al. Genotype and phenotype in patients with Noonan syndrome and a RIT1 mutation. Genet Med. 2016;18(12):1226-34.

Kriz C, Flores S, Villarreal EG, Bronicki RA, Loomba RS. Impact of noonan syndrome on admissions for pediatric cardiac surgery. Minerva Pediatr. 2019;28.

Kuniba H, Pooh RK, Sasaki K, Shimokawa O, Harada N, Kondoh T, et al. Prenatal diagnosis of Costello syndrome using 3D ultrasonography amniocentesis confirmation of the rare HRAS mutation G12D. Am J Med Genet A. 2009; 149A(4):785-7.

Lee KA, Williams B, Roza K, Ferguson H, David $\mathrm{K}$, Eddleman K, et al. PTPN11 analysis for the prenatal diagnosis of Noonan syndrome in $\mathrm{fe}$ tuses with abnormal ultrasound findings. Clin Genet. 2009;75(2):190-4.

Lee WJ, Lee DW, Yang JH, Chang SE, Lee MW, Choi JH, et al. Novel occurrence of microcystic lymphatic malformation and linear portwine stain in patients with Noonan syndrome. Int J Dermatol. 2010;49(2):232-3.

Li D, March ME, Gutierrez-Uzquiza A, Kao C, Seiler C, Pinto E, et al. ARAF recurrent mutation causes central conducting lymphatic anomaly treatable with a MEK inhibitor. Nat Med. 2019;25(7):1116-22.

Lin AE, O'Brien B, Demmer LA, Almeda KK, Blanco CL, Glasow PF, et al. Prenatal features of Costello syndrome: ultrasonographic findings and atrial tachycardia. Prenat Diagn. 2009;29(7):682-90.

Lissewski C, Chune V, Pantaleoni F, De Luca A, Capri Y, Brinkmann J, et al. Variants of SOS2 are a rare cause of Noonan syndrome with particular predisposition for lymphatic complications. Eur J Hum Genet. 2021;29(1):5160.

Lo IF, Brewer C, Shannon N, Shorto J, Tang B, Black G, et al. Severe neonatal manifestations of Costello syndrome. J Med Genet. 2008; 45(3):167-71.

Mathur D, Somashekar S, Navarrete C, Rodriguez MM. Twin infant with lymphatic dysplasia diagnosed with Noonan syndrome by molecular genetic testing. Fetal Pediatr Pathol. 2014; 33(4):253-7.

Menashe M, Arbel R, Raveh D, Achiron R, Yagel $S$. Poor prenatal detection rate of cardiac anomalies in Noonan syndrome. Ultrasound Obstet Gynecol. 2002;19(1):51-5.

Milosavljevic D, Overwater E, Tamminga S, de Boer K, Elting MW, van Hoorn ME, et al. Two cases of RIT1 associated Noonan syndrome: Further delineation of the clinical phenotype and review of the literature. Am J Med Genet A. 2016;170(7):1874-80.

Moola SMZ, Tufanaru C, Aromataris E, Sears K, Sfetcu R. Systematic reviews of etiology and risk. In: Aromataris E, Munn Z, editors. JBI Manual for Evidence Synthesis. JBI; 2020. p. 217-69.

Moore JE, Jr., Bertram CD. Lymphatic System Flows. Annu Rev Fluid Mech. 2018;50:45982.

Morcaldi G, Bellini T, Rossi C, Maghnie M, Boccardo F, Bonioli E, et al. Lymphodysplasia and Kras Mutation: A Case Report and Literature Review. Lymphology. 2015;48(3):121-7.

Mucciolo M, Dello Russo C, D'Emidio L, Mesoraca A, Giorlandino C. Next Generation Sequencing Approach in a Prenatal Case of Cardio-Facio-Cutaneus Syndrome. Int J Mol Sci. 2016;17(6):952.

Rauen KA, Tidyman WE, Estep AL, Sampath S, Peltier HM, Bale SJ, et al. Molecular and functional analysis of a novel MEK2 mutation in cardio-facio-cutaneous syndrome: Transmission through four generations. Am J Med Genet A. 2010;152A(4):807-14.

Romano AA, Allanson JE, Dahlgren J, Gelb BD, Hall B, Pierpont ME, et al. Noonan syndrome: clinical features, diagnosis, and management guidelines. Pediatrics. 2010;126(4): 746-59.

Schlüter G, Steckel M, Schiffmann H, Harms K, Viereck V, Emons G, et al. Prenatal DNA diagnosis of Noonan syndrome in a fetus with massive hygroma colli, pleural effusion and ascites. Prenat Diagn. 2005;25(7):574-6.

Schubbert S, Zenker M, Rowe SL, Böll S, Klein C, Bollag G, et al. Germline KRAS mutations cause Noonan syndrome. Nat Genet. 2006; 38(3):331-6.

Shaw AC, Kalidas K, Crosby AH, Jeffery S, Patton MA. The natural history of Noonan syndrome: a long-term follow-up study. Arch Dis Child. 2007;92(2):128-32.

Sinico M, Bassez G, Touboul C, Cavé H, Vergnaud A, Zirah C, et al. Excess of neuromuscular spindles in a fetus with Costello syndrome: a clinicopathological report. Pediatr Dev Pathol. 2011;14(3):218-23.

Smpokou P, Tworog-Dube E, Kucherlapati RS, Roberts AE. Medical complications, clinical findings, and educational outcomes in adults with Noonan syndrome. Am J Med Genet A. 2012;158A(12):3106-11.

Stuurman KE, Joosten M, van der Burgt I, Elting M, Yntema HG, Meijers-Heijboer H, et al. Prenatal ultrasound findings of rasopathies in a cohort of 424 fetuses: update on genetic testing in the NGS era. J Med Genet. 2018;56(10): 654-61.

Tajan M, Paccoud R, Branka S, Edouard T, Yart A. The RASopathy Family: Consequences of Germline Activation of the RAS/MAPK Pathway. Endocr Rev. 2018;39(5):676-700.

Templin L, Baumann C, Busa T, Heckenroth H, Pouvreau N, Toutain A, et al. Prenatal findings in cardio-facio-cutaneous syndrome. Am J Med Genet A. 2016;170A(2):441-5.

Terry J, Rauen KA, Nowaczyk MJ. Fetal autopsy findings of cardiofaciocutaneous syndrome with a unique BRAF mutation. Pediatr Dev Pathol. 2014;17(1):59-63. 
Thompson D, Patrick-Esteve J, Surcouf JW, Rivera $D$, Castellanos $B$, Desai $P$, et al. RAF1 variants causing biventricular hypertrophic cardiomyopathy in two preterm infants: further phenotypic delineation and review of literature. Clin Dysmorphol. 2017;26(4):195-9.

Van der Burgt I. Noonan syndrome. Orphanet J Rare Dis. 2007;2:4.

Vigneswaran TV, Homfray T, Allan LD, Simpson JM, Zidere V. Persistently elevated nuchal translucency and the fetal heart. J Matern Fetal Neonatal Med. 2018;31(18):2376-80.

Villani A, Greer MC, Kalish JM, Nakagawara A, Nathanson KL, Pajtler KW, et al. Recommendations for cancer surveillance in individuals with RASopathies and other rare genetic conditions with increased cancer risk. Clin Cancer Res. 2017;23(12):e83-90.

Wang N, Shi W, Jiao Y. A PTPN11 mutation in a woman with Noonan syndrome and proteinlosing enteropathy. BMC Gastroenterol. 2020;20(1):34.

Witters I, Denayer E, Brems H, Fryns JP, Legius E. The cardiofaciocutaneous syndrome: prenatal findings in two patients. Prenat Diagn. 2008;28(1):53-5.

Yaoita M, Niihori T, Mizuno S, Okamoto N, Hayashi S, Watanabe A, et al. Spectrum of mutations and genotype-phenotype analysis in Noonan syndrome patients with RIT1 mutations. Hum Genet. 2016;135(2):209-22.
Yoshida R, Miyata M, Nagai T, Yamazaki T, Ogata T. A 3-bp deletion mutation of PTPN11 in an infant with severe Noonan syndrome including hydrops fetalis and juvenile myelomonocytic leukemia. Am J Med Genet A. 2004;128A(1):63-6.

Zenker M, Buheitel G, Rauch R, Koenig R, Bosse $\mathrm{K}$, Kress W, et al. Genotype-phenotype correlations in Noonan syndrome. J Pediatr. 2004;144(3):368-74.

Zmolikova M, Puchmajerova A, Hecht P, Lebl J, Trkova M, Krepelova A. Coarctation of the aorta in Noonan-like syndrome with loose anagen hair. Am J Med Genet A. 2014; 164A(5):1218-21. 